\title{
Distribution of thalamic nociceptive neurons activated from the tail of the rat
}

\author{
Chen-Tung Yen ${ }^{1}$, Tsu-Ching $\mathrm{Fu}^{2,3}$ and Rong-Chi Chen ${ }^{2,3}$ \\ ${ }^{\prime}$ Department of Zoology, College of Science, and Departments of ${ }^{2}$ Physiology and ${ }^{3}$ Neurology, College of Medicine, National \\ Taiwan University, Taipei (Taiwan)
}

(Accepted 28 February 1989)

Key words: Thalamus; Nociceptive neuron; Pain; Tail; Rat; Extracellular single unit recording

\begin{abstract}
The purpose of the present study was to map systematically in the thalamus the distribution of neurons processing nociceptive information from the tail of the rat. Pentobarbital-anesthetized and gallamine-paralyzed rats were used. Glass microelectrodes were used to record extracellularly from thalamic neurons. Noxious radiant heat stimuli were applied to the tail with a tail-flick apparatus, and the recorded neurons were localized with horseradish peroxidase deposits or by marking electrodes left in situ. A number of 121 neurons were tested of which 45 responded. Of these, 13 were located in the ventrobasal complex (VB), 17 were located in the central lateral nucleus and the parafascicular nucleus of the intralaminar nuclei (ILN). The rest of the responding neurons were located in the posterior group, the reticular thalamic nucleus, and the zona incerta. The nucleus submedius was not examined specifically. It is concluded that the VB and the ILN are two of the most important thalamic nuclei for processing nociceptive information from the tail of the rat.
\end{abstract}

\section{INTRODUCTION}

The thalamus, especially its ventrobasal complex (VB), posterior group (PO) and intralaminar nuclei (ILN), are implicated in the processing of nociceptive somatosensory information ${ }^{9,13}$. There are 3 major lines of evidence in support of this statement. First, some thalamic neurons are either selectively or maximally activated by noxious stimuli; second, stimulation of selective points in the thalamus produces painful sensation in human patients or behaviors indicative of pain in experimental animals; and third, selective lesions made in the thalamus alleviate pain in some patients (for reviews, see refs. 2, 6, 9 and 13).

Although the involvement of the thalamus in nociceptive mechanisms has been studied intensively ${ }^{6,13}$, this field is still filled with uncertainties and controversies. For example, although it has been established that the VB is involved in the sensory discriminative aspect of pain ${ }^{12}$, the roles of the PO, the ILN or other thalamic nuclei are still not certain. It is even controversial as to exactly which thalamic nuclei are involved ${ }^{2}$.

Some of the difficulties may be resolved by choosing a suitable animal model. The tail of the rat seems to be an attractive system because it is relatively easy to manipulate owing to its long length, short hair, and simple shape, and because the behavioral consequences of its activation by noxious radiant heat stimuli, i.e. the tail-flick test, have been studied extensively ${ }^{4}$. Noxious radiant heat applied with the tail-flick apparatus is more specific than mechanical noxious stimuli which will simultaneously activate low threshold mechanoreceptors ${ }^{5}$, 10,11. Also, if used properly, a radiant heat stimulus can be applied repetitively, an important requirement for any mapping study, in contrast to more damaging tests such as immersion of the tail in a hot water bath ${ }^{5,7}$. In this paper, we report our prelimi-

Correspondence: C.-T. Yen, Department of Zoology, National Taiwan Unversity, Taipei, Taiwan, R.O.C. 
nary results on the distribution of nociceptive neurons which were activated by noxious radiant heat stimuli applied to the tail in the rat thalamus.

\section{MATERIALS AND METHODS}

Thirty-one male Long-Evans rats, weighing 302$520 \mathrm{~g}$, were used. The animals were anesthetized initially with pentobarbital $(60 \mathrm{mg} / \mathrm{kg}$ i.p.). Supplementary doses $(0.4 \%, 0.5-1 \mathrm{ml}$, i.v.) were given intermittently to suppress flexor reflex and tachycardiac responses produced by noxious stimuli. Rectal temperature of the animal was recorded continuously (Harvard sensortek BAT-12) and maintained between 36 and $38^{\circ} \mathrm{C}$ with an infrared lamp. The animals were paralyzed with gallamine triethiodide (i.v., $20 \mathrm{mg}$ initially and $10 \mathrm{mg}$ every half to one hour) and respired with a rodent ventilator (New England model 131). Expiratory $\mathrm{CO}_{2}$ concentration was monitored continuously with a $\mathrm{CO}_{2}$ monitor (Datex Normocap) and maintaind at $4 \%$.

The rat was mounted on a stereotaxic apparatus (modified from the AB Transvertex system). A craniotomy exposed the cortex directly above the right thalamus. In each animal, a series of recordings were made in the thalamus at one or two transverse planes (P2-P5 in Fig. 3). Within each transverse plane, all recording tracks started from the same point on the surface of the cortex 2-3 mm lateral to the midline with different angles for each track. The angles usually ranged between $10^{\circ}$ laterally to $30^{\circ}$ medially, with $5^{\circ}$ between each tract, and a total of 5-9 recording tracks were made in each transverse plane, thus covering the greater part of the thalamus.

Two types of glass microelectrodes were used to record from thalamic neurons. Microelectrodes of the first type were made from glass tubes with a microfilament inside. They were filled with a solution containing $5 \%$ horseradish peroxidase (HRP, Sigma type VI) in Tris-buffered $0.5 \mathrm{M} \mathrm{KCl}(\mathrm{pH}=$ 7.4). The $D C$ resistances of these electrodes were about $10 \mathrm{M} \Omega$. HRP deposits were made at selected points of the recording track by passing $0.2-0.4 \mu \mathrm{A}$ depolarizing current pulses with a high-voltage electrometer (Eutectic 400B) to mark the recorded sites. The second type of microelectrode was made from glass tubes without a microfilament. Their tips were
A
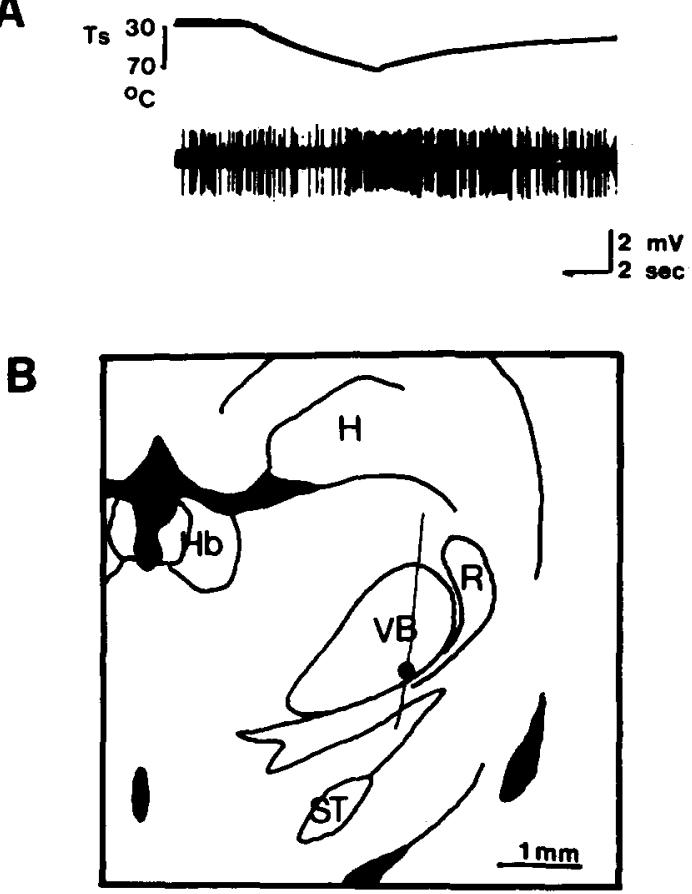

Fig. 1. Example of the response of a thermal nociceptive neuron in the VB to radiant heat applied to a small region of the tail. The receptive field of this neuron was in the middle part of the tail. A: top trace: surface temperature (Ts) of the tail. During the 6-s stimulation period, Ts increased from 28 to $73{ }^{\circ} \mathrm{C}$. Lower trace: VB neuron activity. Notice the sharp increase in spike frequency during the peak of the Ts rise. $B$ : location of the VB neuron ( $)$. Abbreviations are the same as in Fig. 3.

broken to 1-2 $\mu \mathrm{m}$ under a microscope. They were filled with $3 \mathrm{M} \mathrm{NaCl}$ solution. At the end of a series of trackings and recordings, the microelectrode was cut at the shank and left in situ for histological identification.

The tail of the animal was placed on a tail-flick apparatus (EMDIE TF6) for radiant heat stimulation. Specific regions of the tail were stimulated with focused heat radiation. The area radiated was a circle approximately $1 \mathrm{~cm}$ in diameter. The total duration of the radiation was $6 \mathrm{~s}$. Upon turning on the radiant heat lamp, the surface temperature of the skin rose gradually and reached a peak temperature of $70-80^{\circ} \mathrm{C}$ at the end of the 6-s period (measured with a small Harvard IT-21 sensor, Figs. 1 and 2). The peak temperature measured simultaneously under the skin was $20-25^{\circ} \mathrm{C}$ less than the surface temperature and the radiated skin was never more 
than slightly reddish after several exposures. To minimize possible sensitization of the radiated $\mathrm{skin}^{3}$, different points along the caudal one third of the tail starting from caudal and moving to rostral were used for testing each thalamic unit. A thalamic unit was labeled as thermal nociceptive if the unit demonstrated significant response during all $2-4$ trials of the radiant heat tests, repeated after the surface temperature had returned to control values between 28 and $34{ }^{\circ} \mathrm{C}$. Units were also tested for responsiveness to contralateral sciatic nerve stimulation and to mechanical stimuli applied to various parts of the body.

At the end of the recording experiment, the animal was deeply anesthetized with i.v. pentobarbital and perfused intracardially with saline followed by $10 \%$ formalin. Then, the brain was removed and placed in $10 \%$ formalin for further fixation for a week at $4{ }^{\circ} \mathrm{C}$. Fifty $-\mu \mathrm{m}$ thick frozen sections were cut in a cryostat (Reichert-Jung 2800 Frigocut N). In those with HRP deposits, the sections were reacted

\section{A}
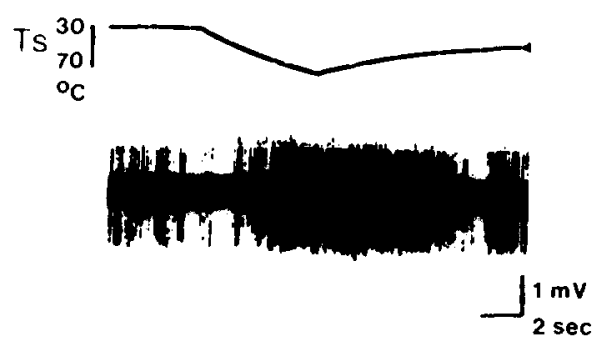

B

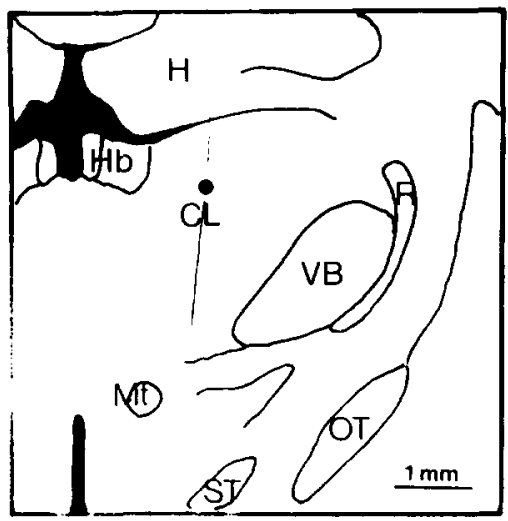

Fig. 2. Example of the responses of a thermal nociceptive neuron in the $\mathrm{CL}$. This neuron responded to pressure and pinch applied to the tail and the ipsilateral limbs. A: surface temperature (Ts) of the tail was raised from 31 to $77^{\circ} \mathrm{C}$. The activity of the $\mathrm{CL}$ neuron increased during the peak Ts. B: location of the $\mathrm{CL}$ neuron (O). Abbreviations are the same as in Fig. 3. with cobalt-intensified diaminobenzidine $(\mathrm{DAB})^{1}$. Otherwise, Cresyl violet staining was used. Camera lucida drawings of the stained sections were made with a drawing tube attached to a stereomicroscope (Wild M3). Location of the recorded units were identified by the brown black DAB reaction product (approximately $200 \mu \mathrm{m}$ in diameter), or by extrapolation to Nissl-stained sections from the depths recorded during the experiment.

\section{RESULTS}

A number of 121 thalamic units were tested by the radiant heat stimuli; 45 responded.

In Figs. 1 and 2, two representative examples of the responses of thalamic thermal nociceptive units are shown. The response to radiant heat stimulus applied with the tail-flick apparatus of a unit located in the ventrolateral edge of the $\mathrm{VB}$ is shown in Fig. 1. This VB unit had an ongoing activity. Upon turning on the radiant lamp, there seemed to be a slight decrease in unit activity at first. However, when skin temperature exceeded $60^{\circ} \mathrm{C}$, this unit started to fire vigorously. This response subsided within a few seconds. The responses of another unit located in the central lateral nucleus (CL) of the ILN are shown in Fig. 2. This CL unit also was spontaneously active. The response of this $\mathrm{CL}$ unit to noxious thermal stimulation of the tail was brisk.

The responses of all nociceptive thalamic units were clearly excitatory; significant inhibition was not observed. No special test was made, however, to examine inhibition in silent units. These units were judged to be recorded from the somatodendritic area of the neuron because the action potentials were biphasic, with long duration ( $>1 \mathrm{~ms}$ ), and high amplitude (usually $2-10 \mathrm{mV}$ ) spikes.

The location of these 45 thermal nociceptive thalamic neurons and the locations of the other 76 cells whose activities were not changed by thermal tail stimulation are shown in Fig. 3. Nociceptive thalamic neurons were concentrated in the lateral and dorsal part of the VB $(13,29 \%)$, and in the ILN $(17,38 \%)$, especially the central lateral (CL) and the parafascicular (PF) nuclei. Scattered thermal nociceptive neurons were also found in the $\mathrm{PO}$, the reticular nucleus (R), the $\mathrm{ZI}$, and the basal ganglia (BG). 

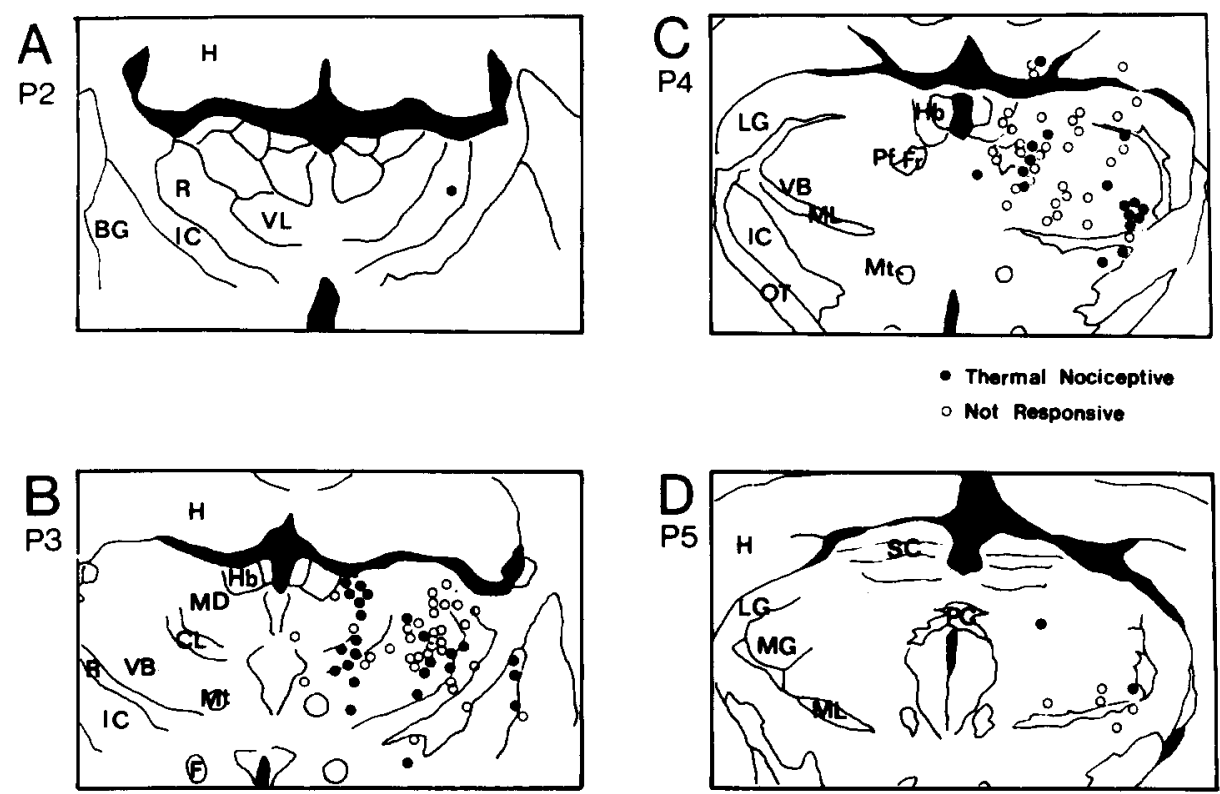

Fig. 3. Locations of the radiant positive $(O)$ and negative $(O)$ neurons in the diencephalon. Drawings of transverse sections of the rat brain modified from the atlas of Paxinos and Watson ${ }^{8}$. The 4 sections were the levels of $2 \mathrm{~mm}(\mathrm{~A}), 3 \mathrm{~mm}(\mathrm{~B}), 4 \mathrm{~mm}(\mathrm{C})$ and $5 \mathrm{~mm}$ (D) posterior to bregma. BG, basal ganglia; H, hippocampus; Hb, habenula; CL, central lateral nucleus; F, fornix; Fr, fasciculus retroflexus; IC, internal capsule; LG, lateral geniculate body; MD, mediodorsal nucleus; MG, medial geniculate body; ML, mediolemniscal tract; Mt, mamillothalamic tract; PG, posterior commissure; Pf, parafascicular nuclei; OT, optic tract; R, thalamic reticular nucleus; SC, superior colliculus; ST, subthalamic nucleus; VB, ventrobasal nuclei; VL, ventrolateral nuclei.

\section{DISCUSSION}

In this study, nociceptive thalamic neurons responding to radiant heat stimuli applied on the tail of the rat were systematically mapped. They were found mainly in the VB and in CL and PF of the ILN.

The fact that there are neurons in the thalamus maximally activated by noxious stimuli has been established in the monkey, the cat and the rat (for review see ref. 13). In the rat, pinching the tail or dipping the tail into hot water have been shown to activate neurons in the $\mathrm{VB}$, the $\mathrm{PO}$ and the ILN $^{5,7,10,11}$. These studies, however, dealt only with the $\mathrm{VB}^{5,7}$, or studied nociceptive thalamic neurons in relation to the whole body ${ }^{10,11}$. In this report, we narrowed our objective to study only those central neurons related to the tail using a selective noxious stimulus. We specifically addressed the question: which parts of the thalamus could be activated by noxious stimulation of the tail of the rat. Qualitatively, our results are similar to those of Mitchell and Hellon ${ }^{7}$, Guilbaud et al. ${ }^{10}$, and Peschanski et al. ${ }^{11}$ in that thalamic nociceptive neurons representing the tail could be found in the VB, PO and ILN. In addition, we demonstrated with evenly distributed sampling, that quantitatively, most of these neurons were located in the VB and the ILN. A few of them were found in the lateral part of the $\mathrm{ZI}$ and the dorsal caudal part of the globus pallidus. With either the simple tail-flick stimulation alone or in combination with electrical stimulation of the sciatic nerve and simple mechanical stimulation of the body, we could not find a significant difference between the responsive properties of VB neurons and those of ILN neurons. The only notable distinction was that nociceptive neurons with small receptive field (part of the tail) were restricted to the VB. In all other nuclei, neurons responded to stimulation of at least the whole tail and a part of the hindbody, and sometimes responded to stimulation of the entire body. This may indicate that the VB is more implicated in the localization of noxious stimuli. Further study and more qualitative and quantitative analysis will be needed to reveal any subtle differences among neurons in different thalamic nuclei. 


\section{ACKNOWLEDGEMENTS}

We are grateful for the excellent technical assistance of Mr. Wann-Jer Jong and Mr. Yu-Chiunn Lai. We are also grateful to Drs. C.N. Honda and E.G.

\section{REFERENCES}

1 Adams, J.C., Technical considerations in the use of horseradish peroxidase as a neuronal marker, Neuroscience, 2 (1977) 141-146.

2 Albe-Fessard, D., Berkeley, K.J., Kruger, L., Ralston III, H.J. and Willis Jr., W.D., Diencephalic mechanisms of pain sensation, Brain Res. Rev., 9 (1985) 217-296.

3 Besson, J.-M. and Chaouch, A., Peripheral and spinal mechanisms of nociception, Physiol. Rev., 67 (1987) 67-186.

$4 \mathrm{Fu}$, T.C. and Dewey, W.L., Morphine antinociception: evidence for the release of endogenous substances, Life Sci., 25 (1979) 53.

5 Guilbaud, G., Peschanski, M., Gautron, M. and Binder, D., Neurons responding to noxious stimulation in VB complex and caudal adjacent regions in the thalamus of the rat, Pain, 8 (1980) 303-318.

6 Jones, E.G., The Thalamus, Plenum, New York, 1985.

7 Mitchell, D. and Hellon, R.F., Neuronal and behavioral responses in rats during noxious stimulation of the tail,
Jones for their advice in preparing the manuscript. This project was supported by Grant NSC 760412-B002-13 from the National Science Council of the Republic of China.

Proc. R. Soc. Lond. Ser. B, 197 (1977) 169-194.

8 Paxinos, G. and Watson, C., The Rat Brain in Stereotaxic Coordinates, 2nd edn., Academic, Sydney.

9 Perl, E.R., Pain and nociception. In I. Darian-Smith (Ed.), Handbook of Physiology, Section I, Vol. III, American Society of Physiology, 1984, pp. 915-975.

10 Peschanski, M., Guilbaud, G., Gautron, M. and Besson, J.-M., Encoding of noxious heat messages in neurons of the ventrobasal thalamic complex of the rat, Brain Research, 197 (1980) 401-413.

11 Peschanski, M., Guilbaud, G. and Gautron, M., Posterior intralaminar region in rat: neuronal responses to noxious and non-noxious cutaneous stimuli, Exp. Neurol., 72 (1981) 226-238.

12 Price, D.D. and Dubner, R., Neurons that subserve the sensory-discriminative aspects of pain, Pain, 3 (1977) 307-338.

13 Willis Jr., W.D., The Pain System, the Neural Basis of Nociceptive Transmission in the Mammalian Nervous System, Karger, New York, 1985. 